\title{
Teaching an old dog new tricks: potential antiatherothrombotic use for statins
}

\author{
Roy L. Silverstein \\ Medical College of Wisconsin, Milwaukee, Wisconsin, USA.
}

\begin{abstract}
Thrombotic complications represent a highly significant component of morbidity and mortality associated with hypercholesterolemia and atherosclerosis. In this issue of the JCI, Owens et al. report possible mechanisms underlying the prothrombotic, proinflammatory state accompanying hypercholesterolemia. Using rodent, monkey, and human subjects, they show that circulating oxidized LDL and circulating monocyte-derived tissue factor are important instigating factors driving the thrombotic, inflammatory phenotype and, surprisingly, that statin therapy ameliorated the phenotype even in the absence of lowering cholesterol levels. The studies raise the intriguing possibility that therapies directed at pathways generating oxidant stress or pathways involved in transmitting oxidized LDL-mediated signals in circulating platelets and monocytes could have antiatherothrombotic potential, probably with minimal anticoagulant and hemorrhagic potential.
\end{abstract}

Morbidity and mortality from atherosclerosis, including acute myocardial infarction, sudden death, stroke, and limb loss, put an enormous burden on health care systems. Hypercholesterolemia is a major risk factor for atherosclerosis, which is caused by the progressive formation of arterial plaques that are characterized by accumulation of lipids, in particular cholesterol and its derivatives, and inflammatory cells. Most of the severe clinical events derive from acute or subacute thrombosis at a site of an unstable, inflamed, or ruptured atherosclerotic plaque or at a site of recent mechanical or pharmacologic intervention. Available pharmacologic approaches to prevent or treat these devastating thrombotic events are mainly limited to antiplatelet agents, including aspirin and P2Y12 ADP receptor antagonists, and/or anticoagulants. Resistance to these agents and/or intolerance related to hemorrhagic side effects are significant problems for many patients. Therefore, identification of new therapeutic targets is desperately needed. In an elegant series of studies reported on in this issue of $J C I$, Owens and colleagues generated data that raise the prospect of using an "old" drug (a statin) to target "new" pathways to blunt the prothrombotic state associated with advanced atherosclerosis (1).

Conflict of interest: The author has declared a conflict of interest with Pfizer.

Citation for this article: J Clin Invest. 2012; 122(2):478-481. doi:10.1172/JCI61857.

\section{Hypercholesterolemia triggers crosstalk between proinflammatory pathways and the coagulation cascade}

In addition to directly promoting atherosclerotic plaque formation, hypercholesterolemia is also associated with platelet hyperactivity, although the mechanisms by which hypercholesterolemia produces a procoagulant state remain incompletely understood. The work of Owens and colleagues (1) confirms an association between hypercholesterolemia and a proinflammatory, prothrombotic state in a cohort of human subjects with familial hypercholesterolemia, nonhuman primates with diet-induced hypercholesterolemia, and LDL receptor-deficient mice with Western diet-induced atherosclerosis. It also delineates an underlying mechanism.

Owens et al. observed that levels of circulating biomarkers for activation of coagulation, including thrombin-antithrombin complexes, d-dimer, and monocyte-derived tissue factor-expressing microparticles $\left(\mathrm{TF}^{+} \mathrm{MP}\right)$, were uniformly elevated in the human subjects and in both animal models, as were markers for inflammation, including high-sensitivity C-reactive protein (hsCRP) and IL-6. In the mouse model, they validated the significance of the elevated levels of coagulation biomarkers by showing that experimentally induced arterial thrombosis in vivo was enhanced when compared with that in control mice. Using infusion of an inhibitory TF-specific monoclonal antibody and transplantation of bone marrow from mice genetically deficient in TF, they showed that the driving force for the hypercholesterolemia-associated prothrombotic phenotype, at least in the mice, was the generation of $\mathrm{TF}^{+} \mathrm{MP}$. Interestingly, the proinflammatory phenotype was also ameliorated by blocking or deleting TF, demonstrating important crosstalk between the coagulation and inflammation systems.

\section{Oxidant stress and oxidized LDL drive atherothrombosis}

A unifying principle in all three "models" is most likely ongoing chronic oxidant stress; in all settings, the authors documented the presence of oxidized LDL (oxLDL) in the circulation. Oxidant stress within the vessel wall has long been considered a key pathophysiological instigator of atherogenic processes (ref. 2; as shown in Figure 1). Transudation and entrapment of LDL particles and monocytes into the subendothelial space occur early in atherogenesis. ROS generated by the inflammatory milieu within the atherogenic vessel wall then modify the LDL by oxidizing both protein and lipid moieties, creating what are called collectively oxLDL (3). OxLDL are recognized by scavenger receptors on macrophages, particularly CD36, which initiate a cascade of events, including internalization of the bound oxLDL, activation of proinflammatory pathways, and activation of transcriptional pathways (4). These result in a feed-forward loop that increases leukocyte recruitment into the vessel wall, inhibits macrophage migration out of the vessel wall (5), increases ROS generation while suppressing natural antioxidant defense pathways (thus increasing oxLDL formation) (6), and increases CD36 expression (7). The net effect is formation and accumulation of lipid-laden foam cells and proinflammatory immune cells, which together form plaque (Figure 1). 


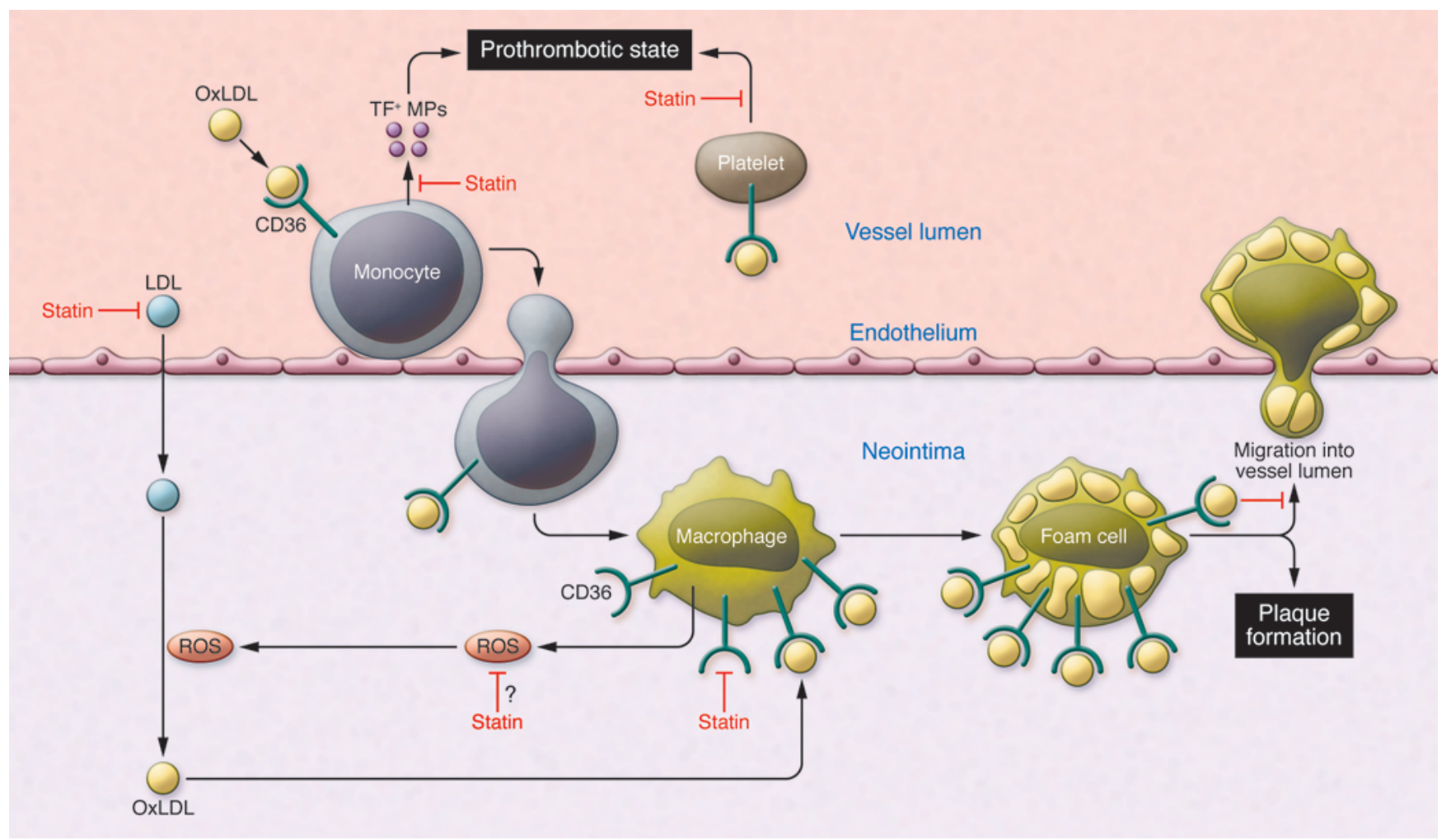

\section{Figure 1}

Atherothrombotic processes occur both within the vessel wall (neointima) and in the circulation. In response to injury or inflammation, LDL and monocytes enter the vessel wall and become trapped. Monocytes differentiate into inflammatory macrophages that produce ROS, which oxidize trapped LDL particles to form oxLDL. These then interact with macrophages through scavenger receptors, such as CD36, activating signaling pathways that lead to enhanced inflammation and ROS generation, foam cell formation, and entrapment of the foamy macrophages to form plaque. OxLDL in the circulation interacts with blood monocytes and platelets, through CD36 and other receptors, leading to the generation of $\mathrm{TF}^{+} \mathrm{MPs}$ and hyperreactive platelets. These create a circulating "prothrombotic state" that increases risk of acute thrombosis in the setting of unstable plaque. Statins have the potential to interrupt these processes at multiple levels by lowering circulating LDL levels, downregulating monocyte/macrophage CD36 expression, and inhibiting ROS generation and proinflammatory signaling.

Although abundant data from animal models and correlative human studies support this oxidative stress hypothesis $(8,9)$, there has been considerable controversy and disbelief by some investigators, mainly because large and medium-sized interventional trials of antioxidant therapy in humans have generally failed to prevent the complications of atherosclerosis (3). In my mind, however, these trials have been flawed by poor choice of antioxidants (some vitamin E formulations, for example, can actually promote oxidative stress) and lack of convincing evidence that the tested "antioxidant" therapies actually targeted the relevant vascular or circulating oxidant pathways.

Owens et al. described several interventions that reversed the prothrombotic phenotype in hypercholesterolemic individuals (1). In the human studies, they showed that apheresis dramatically lowered all of the proinflammatory and prothrombotic biomarkers. This is consistent with a direct mechanistic role for oxLDL in triggering the prothrombotic phenotype, since the process removes circulating oxLDL. However, it is also possible that the efficacy of apheresis may relate to downregulation of a humoral immune response to oxLDL, which data suggest may be proatherogenic (10). Nevertheless, the studies suggest that targeting circulating oxLDL in hypercholesterolemic individuals may be worthwhile, as it appears that oxLDL interactions with circulating platelets and monocytes may be as important as oxLDL interaction with cells within the vessel wall in manifesting the morbidity and mortality from atherosclerosis. The authors suggest, based on their in vitro and mouse studies, that oxLDL-mediated generation of $\mathrm{TF}^{+}$ MP from circulating monocytes drives both the prothrombotic and proinflammatory phenotypes. It is likely, however, that more complex systems are operating in humans.

\section{Statin therapy interrupts the proinflammatory, atherothrombotic phenotype associated with hypercholesterolemia}

Remarkably, in both mice and monkeys, Owens et al. found that a short course of simvastatin dramatically lowered levels of the proinflammatory and prothrombotic biomarkers without affecting levels of cholesterol (1). That statins may have antiatherothrombotic effects independent of their ability to lower cholesterol levels is not a new concept, and the efficacy of simvastatin in the studies of Owens et al. is unlikely to be an unusual off-target effect of the specific statin. The JUPITER clinical trial, for example, showed that rosuvastatin therapy decreased cardiovascular events in patients with elevated hsCRP but normal levels of LDL (11), and animal studies have shown various statins have antiatherothrombotic benefits that are not simply explainable by lowering 
levels of LDL. The pleiotropic effects of statins are well documented (12); inhibiting HMG CoA reductase (the target of statins) prevents formation of isoprenyl metabolic intermediates as well as cholesterol and thus blocks posttranslational prenylation of important signaling proteins, including small molecular weight G proteins. G protein signaling is critical for generating ROS and proinflammatory and platelet-activating pathways. Statins also downregulate CD36 expression on monocytes in diabetic patients (13) and activate specific transcriptional pathways, including those mediated by Krüppel-like factors (14), which may have atheroprotective effects.

\section{Scavenger receptor CD36 links oxidant stress to atherothrombosis}

While the work of Owens et al. provides important new insights into the mechanistic basis of the prothrombotic state accompanying atherosclerosis and hypercholesterolemia (1), it has not answered all the questions, and, as with all good research, it has raised new ones. Among the most clinically relevant is whether it is rational to consider statins as a "new" class of antithrombotic agents - one that has no anticoagulant activity and therefore little or no hemorrhagic potential. It also behooves us to ask whether generation of $\mathrm{TF}^{+} \mathrm{MP}$ represents the "whole story" of the mechanistic basis of the prothrombotic state accompanying atherosclerosis and hypercholesterolemia. Emerging data suggest that oxLDL signaling via CD36 on platelets and other vascular cells may be an important component of the hypercholesterolemia-mediated prothrombotic state (refs. 15, 16; Figure 1) and that classes of MP that do not express $\mathrm{TF}$, but that interact with platelet CD36 via surface exposure of oxidized phospholipids, may also contribute to thrombosis $(6,17)$. The studies of Owens et al. certainly point to an important role for $\mathrm{TF}^{+} \mathrm{MP}$, but only a small subset of circulating MP generated during chronic inflammatory conditions, such as metabolic syndrome, acute coronary syndrome, cancer, and obesity, express TF. Thus, a better understanding of scavenger receptor-mediated signaling, MP-generating pathways, and prothrombotic influences of TF-negative MP could lead to the discovery of novel specific targets for atherothrombosis.

Genome-wide association studies have linked CD36 genetic polymorphisms to risk of myocardial infarction and sudden death in patients with atherosclerotic coronary diseases (18). Polymorphisms have also been associated with levels of expression of CD36 on platelets and monocytes $(19,20)$, suggesting that genetic influences may "titer" prothrombotic and proatherogenic cellular responses to oxLDL. Interestingly, human CD36 deficiency is common; nearly $1 \%$ of individuals of African or Asian descent are homozygous for CD36null mutations. These individuals have no obvious phenotype, suggesting that CD36 would be a safe pharmacologic target. Furthermore, inhibition or genetic deletion of mediators of downstream signaling pathways triggered by oxLDL, including specific Src family kinases, MAP kinases, and Vav family guanine nucleotide-exchange factors, blunts CD36-mediated prothrombotic platelet responses and proatherogenic macrophage responses in vitro and in mouse models $(4,21,22)$. Particularly exciting is the emerging concept that many signals emanating from CD36 require the recruitment of specific innate immune system receptors as signaling partners $(23,24)$. In the work of Owens et al., both TLR4 and TLR6 were shown to participate in oxLDL induction of monocyte TF and $\mathrm{TF}^{+} \mathrm{MP}$ in vitro and in oxLDL induction of the prothrombotic, proinflammatory phenotype in vivo (1). Thus, targeting TLRs and/or TLR signaling pathways may have as much relevance to atherothrombosis as to innate immune disorders.

\section{Summary and conclusions}

The elegant work reported by Owens et al. (1) used a combination of rodent and nonhuman primate models along with careful assessment of human subjects with familial hypercholesterolemia to demonstrate that activation of proinflammatory pathways and the coagulation cascade are associated with hypercholesterolemia. Elevated levels of $\mathrm{TF}^{+} \mathrm{MP}$ derived from circulating monocytes were shown to be important participants in promoting both inflammation and thrombosis and, remarkably, treatment of the animals with short courses of simvastatin ameliorated both the prothrombotic and proinflammatory phenotype, despite not lowering cholesterol. These data suggest that statins have important protective effects independent of their ability to lower LDL, perhaps explaining in part their efficacy in preventing cardiovascular complications in patients with "normal" LDL levels in the setting of chronic inflammation. These studies also identify several important targets for potential therapeutic intervention that might not have the bleeding risks associated with current antiplatelet therapies or the myopathies associated with statins. For example, lowering circulating oxLDL levels by properly targeted antioxidants might prevent monocyte activation, TF expression, and MP generation. Similarly, blocking oxLDL-induced monocyte and platelet signaling (via CD36) might inhibit the mechanisms responsible for the atherothrombotic phenotype.

\section{Acknowledgments}

NIH grants P50 HL81011 and P01 HL087018 to Roy L. Silverstein supported the studies referred to in this manuscript related to CD36.

Address correspondence to: Roy L. Silverstein, John and Linda Mellowes Professor and Chair, Department of Medicine, Medical College of Wisconsin, 9200 W. Wisconsin Ave., Suite C5038, Milwaukee, Wisconsin 53226, USA. Phone: 414.805.0518; Fax: 414.805.0524; E-mail: rsilverstein@mcw.edu.

1. Owens AP III, et al. Monocyte tissue factor-dependent activation of coagulation in hypercholesterolemic mice and monkeys is inhibited by simvastatin. J Clin Invest. 2012;122(2):558-568.

2. Rocha VZ, Libby P. Obesity, inflammation, and atherosclerosis. Nat Rev Cardiol. 2009;6(6):399-409.

3. Chisolm GM, Steinberg D. The oxidative modification hypothesis of atherogenesis: an overview. Free Radic Biol Med. 2000;28(12):1815-26.

4. Silverstein RL, Febbraio M. CD36, a scavenger receptor involved in immunity, metabolism, angiogenesis, and behavior. Sci Signal. 2009;2(72):re3.

5. Park YM, Febbraio M, Silverstein RL. CD36 modulates migration of mouse and human macrophages in response to oxidized LDL and contributes to macrophage trapping in the arterial intima. J Clin Invest. 2009;119(1):136-145.

6. Li W, Febbraio M, Reddy SP, Yu D-Y, Yamamoto $\mathrm{M}$, Silverstein RL. CD36 participates in a signaling pathway that regulates reactive oxygen species formation by vascular smooth muscle cells. J Clin Invest. 2010;120(11):3996-4006.

7. Tontonoz P, Nagy L, Alvarez JG, Thomazy VA, Evans RM. PPARgamma promotes monocyte/ macrophage differentiation and uptake of oxidized LDL. Cell. 1998;93(2):241-252.

8. Febbraio M, et al. Targeted disruption of the Class B scavenger receptor, CD36, protects against atherosclerotic lesion development in mice. J Clin Invest. 2000;105(8):1049-1056.

9. Manning-Tobin JJ, et al. Loss of SR-A and CD36 activity reduces atherosclerotic lesion complexity without abrogating foam cell formation in hyperlipidemic mice. Arterioscler Thromb Vasc Biol. 2009;29(1):19-26.

10. Shaw PX, et al. Human-derived anti-oxidized LDL autoantibody blocks uptake of oxidized LDL by macrophages and localizes to atherosclerotic lesions in vivo. Arterioscler Thromb Vasc Biol. 2001; 21(8):1333-1339

11. Ridker PM, et al. Rosuvastatin to prevent vascular events in men and women with elevated C-reactive 
protein. NEngl J Med. 2008;359(21):2195-2207.

12. Mihos CG, Salas MJ, Santana O. The pleiotropic effects of the hydroxy-methyl-glutaryl-CoA reductase inhibitors in cardiovascular disease: a comprehensive review. Cardiol Rev. 2010;18(6):298-304.

13. Mandosi E, et al. Atorvastatin downregulates monocyte CD36 expression, nuclear NFkappaB and TNFalpha levels in type 2 diabetes. J Atheroscler Thromb. 2010;17(6):539-545.

14. Tuomisto TT, et al. Simvastatin has an anti-inflammatory effect on macrophages via upregulation of an atheroprotective transcription factor, Kruppellike factor 2. Cardiovasc Res. 2008;78(1):175-184.

15. Podrez EA, et al. Platelet CD36 links hyperlipidemia, oxidant stress and a pro-thrombotic phenotype. Nat Med. 2007;13(9):1086-1095.

16. Korporaal SJ, et al. Platelet activation by oxidized low density lipoprotein is mediated by CD36 and scavenger receptor-A. Arterioscler Thromb Vasc Biol. 2007;27(11):2476-2483.

17. Ghosh A, Li W, Febbraio M, Espinola RG, McCrae $\mathrm{K}$, Silverstein RL. Platelet CD36 mediates interactions with endothelial cell-derived microparticles and contributes to thrombosis in vivo. J Clin Invest. 2008;118(5):1934-1943.

18. Knowles JW, et al. Association of polymorphisms in platelet and hemostasis system genes with acute myocardial infarction. Am Heart J. 2007; 154(6):1052-1058.

19. Love-Gregory L, et al. Common CD36 SNPs reduce protein expression and may contribute to a protective atherogenic profile. Hum Mol Genet. 2011; 20(1):193-201.

20. Ghosh A, et al. Platelet CD36 surface expression levels affect functional responses to oxidized LDL and are associated with inheritance of specific genetic polymorphisms. Blood. 2011;117(23):6355-6366.

21. Rahaman SO, Lennon DJ, Febbraio M, Podrez EA, Hazen SL, Silverstein RL. CD36-dependent activation of c-Jun $\mathrm{N}$-terminal kinase by oxidized LDL is required for macrophage foam cell formation. Cell Metab. 2006;4(3):211-221.

22. Chen K, Li W, Major J, Febbraio M, Silverstein RL. Vav guanine nucleotide exchange factors link hyperlipidemia and a prothrombotic state. Blood. 2011;117(21):5744-5750

23. Seimon TA, et al. Atherogenic lipids and lipoproteins trigger CD36-TLR2-dependent apoptosis in macrophages undergoing endoplasmic reticulum stress. Cell Metab. 2010;12(5):467-482.

24. Stewart CR, et al. CD36 ligands promote sterile inflammation through assembly of a Toll-like receptor 4 and 6 heterodimer. Nat Immunol. 2010; 11(2):155-161

\title{
Plasmacytoid dendritic cells lead the charge against tumors
}

\author{
Sonia Jiménez-Baranda, ${ }^{1}$ Inês Pires Silva, ${ }^{1,2,3}$ and Nina Bhardwaj ${ }^{1}$
}

\author{
${ }^{1}$ New York University Langone Medical Center and New York University Cancer Institute, New York, New York, USA. \\ 2Instituto Português de Oncologia de Lisboa Francisco Gentil, Lisbon, Portugal. ${ }^{3}$ Programme for Advanced Medical Education, Lisbon, Portugal.
}

\begin{abstract}
Imiquimod is a TLR agonist that is used as an antitumor agent, mainly against skin tumors. Its clinical benefits are well described in several studies; however, the mechanisms behind its antitumor effects are not completely understood. In this issue of the JCI, Drobits and colleagues demonstrate that topical application of imiquimod suppresses cutaneous melanoma by TLR7-dependent recruitment and transformation of plasmacytoid dendritic cells into killer cells; this occurs independently of conventional adaptive immune mechanisms.
\end{abstract}

Imiquimod is a synthetic imidazoquinoline with demonstrable antiviral and antitumor properties. It is formulated as a $5 \%$ cream and has been approved to treat certain types of actinic keratoses, which are premalignant, flat, scaly growths on the skin caused by too much sun exposure; some primary skin malignancies, including basal cell carcinoma (BCC) and squamous cell carcinoma (1); and external genital warts. The efficacy of imiquimod as a treatment for BCC is impressive: complete histological clearance is achieved in almost $80 \%$ of the cases (2). Imiquimod has also been used off-label to treat cutaneous melanoma and locally recurrent mucosal melanoma (3), although it is a less effective treatment for this primary skin malignancy than it is for BCC (4).

Conflict of interest: The authors have declared that no conflict of interest exists.

Citation for this article: J Clin Invest. 2012; 122(2):481-484. doi:10.1172/JCI61345.
Imiquimod modulates diverse immune responses through TLR7 ligation

The antitumor effect of imiquimod is multifactorial. It has direct antiangiogenic (5) and caspase-mediated proapoptotic activity (6). However, it also acts as an "immune response modifier," modulating the function of immune cells, especially antigenpresenting cells such as conventional dendritic cells (cDCs), plasmacytoid dendritic cells (pDCs), monocytes, and macrophages. The effects of imiquimod on immune cells are mediated via activating signals initiated by its binding to TLR7 and, in some instances, to TLR8 (4).

TLRs comprise a family of conserved, germline-encoded pattern recognition receptors that recognize diverse molecules from pathogens and are essential for host defense (7). Upon ligand binding, TLRs recruit an adaptor molecule, most frequently MyD88, and promote activation of signaling molecules and pathways (including the NF- $\mathrm{KB}$ and MAPK pathways) that lead to the production of proinflammatory cytokines (e.g., TNF and IL-12). In pDCs, activation of the transcription factor IRF7 after TLR7 ligation leads to MyD88-dependent production of type I IFNs and upregulation of TNF-related apoptosisinducing ligand (TRAIL) and granzyme B (7), which confer on the pDCs a death-inducing effector function. IL-1 $\beta$ and IL-18 are also released through TLR-independent inflammasome activation (7). Imiquimod can directly (via a TLR) or indirectly (via the type I IFNs derived from imiquimod-stimulated pDCs) stimulate $\mathrm{NK}$ cells, $\mathrm{CD}^{+} \mathrm{T}$ cells, $\mathrm{CD} 4^{+}$ $\mathrm{T}$ cells, and B cells. Agonists of TLRs, including imiquimod, can also reverse $\mathrm{CD}^{+}$Treg function in a TLR8 signaling-dependent, DC-independent manner (8).

As a result of their multifactorial antitumor effects, TLR agonists such as imiquimod are being widely tested as adjuvant therapies in cancer patients. Their administration has been associated with intratumoral infiltration of multiple subsets of DCs, and, when delivered in combination with tumor-associated antigens in humans, they have resulted in delayed time to tumor recurrence (4). However, TLRactivated $\mathrm{cDCs}$ and $\mathrm{pDCs}$ can also induce and/or recruit immune suppressive Tregs in vitro and in vivo, and high levels of pDCs in the tumor microenvironment are associated with poor prognosis (9), indicating that we are far from understand- 